\title{
Human papillomavirus (HPV) and Epstein- Barr virus (EBV) in keratinizing versus non- keratinizing squamous cell carcinoma of the oropharynx
}

\author{
Francesco Broccolo ${ }^{1,5^{*}}$ (D), Giulia Ciccarese ${ }^{2}$, Agostino Rossi ${ }^{3}$, Luca Anselmi ${ }^{4}$, Francesco Drago ${ }^{2}$
} and Antonio Toniolo ${ }^{3}$

\begin{abstract}
Background: Oral and oropharyngeal squamous cell carcinomas (OSCC and OPSCC) represent the majority of head and neck squamous cell carcinomas (HNSCC). Human papillomavirus (HPV) is an important etiologic factor together with Epstein-Barr virus (EBV). Little is known on the prevalence of major herpesviruses [EBV, cytomegalovirus (CMV) and HHV-6, -7 and -8$]$ in HNSCCS.

Methods: Fifty-one formalin-fixed paraffin-embedded (FFPE) tissue samples taken at surgery (40 oropharyngeal, 11 oral) were analyzed for $40 \mathrm{HPV}$ genotypes (20 high-risk types), EBV, CMV, HHV-6, -7 and -8 by quantitative PCR. Expression of the HPV-induced p $16^{\mathrm{INK} 4 \mathrm{~A}}$ protein was also investigated by immunohistochemistry (IHC).

Results: In SCC, the prevalence of EBV was significantly higher compared to that of HPV (EBV 51\% vs. HPV 19.5\%; $P=0$. 005). HPV infection was found in 25\% of OPSCC and in none of the OSCC; conversely, higher prevalence of EBV was found in OSCC (72.7\%). HPV and EBV co-infection was detected only in 4 (10\%) OPSCC. CMV was detected in only two cases, whereas HHV-6, -7 and -8 resulted negative. The prevalence of HPV but no EBV was associated with the nonkeratinizing SCC type (NKSCC) compared to the keratinizing SCC type (KSCC)(HPV-DNA $P<0.005$; EBV = 0.054).
\end{abstract}

Conclusions: Single HPV or EBV positivity was higher in OSCC than in OPSCC. Other potentially oncogenic herpesvirus types were minimally or not represented.

Keywords: EBV, HPV, Oral, Oropharyngeal, Cancer, HPV-induced p16 $6^{\text {INK4A }}$ immunohistochemistry, Co-infection

\section{Background}

Head and neck squamous cell carcinomas (HNSCC) account for approximately $5 \%$ of all cancers and are a serious public health problem worldwide [1]. HNSCC comprise a large group of tumors classified as oropharyngeal squamous cell carcinomas (OPSCC) and oral squamous cell carcinomas (OSCC). HNSCC is etiologically linked to tobacco and/or alcohol consumption [2]. Although chemical risk factors have been widely studied, research revealed that human papillomaviruses (HPV)

\footnotetext{
* Correspondence: francesco.broccolo@unimib.it

${ }^{1}$ Department of Medicine and Surgery (School of Medicine), University of Milano-Bicocca, Monza, Italy

${ }^{5}$ Laboratory of Medical Microbiology, University of Milano-Bicocca, Via

Cadore 48, 20900 Monza, Italy

Full list of author information is available at the end of the article
}

do play an important role in a subset of HNSCC [3]. Currently, $20-30 \%$ of patients with OPSCC do not have the traditional risk factors of smoking and alcohol use and HPV appears as the major driver of malignant transformation [4]. Case-control studies reported a consistent association of HPV infection with HNSCC, particularly for OPSCC $[2,5,6]$. In contrast, the association of HPV with oral and laryngeal cancers has been inconsistent, possibly due to tumor misclassification and the influence of confounding factors like tobacco and alcohol consumption [4]. Exposure to HPV in the head and neck region is associated with sexual behaviors that increase the risk of HNSCC [7]. The association between HPV infection and head and neck carcinogenesis is based on histological similarities between the anogenital epithelium 
and the squamous epithelium of the head and neck region, as well as on the detection of most common oncogenic HPV genotypes in both cervical cancer and HNSCC [8]. Based on epidemiologic observations, 12.8 to 59.9\% HNSCCs are associated with HPV infection [9]. In Europe, the estimated prevalence of HPV in these tumors is approximately $41 \%$ [10]. Limited data are available for Italy regarding HPV detection in HNSCCs, with prevalence ranging from 10 to $46 \%[11,12]$.

The Epstein-Barr virus (EBV) has long been related to nasopharyngeal carcinoma [13]. A recent meta-analysis show a significant association between EBV and OSCCs [14] though there are studies not reporting a significant association [15]. Some members of Herpesviridae family (EBV and HHV-8) are recognized as carcinogens. Other herpesviruses such as cytomegalovirus (CMV) and HHV-6 and - 7 may be associated with a malignant phenotype, but their carcinogenic role remains unclear [16]. Persistent infection with EBV has been linked to the development of malignancies including HPV-associated oral carcinoma. However, the possible role of EBV and other herpesviruses in HPV-associated oral cancers is still poorly understood. The majority of studies were based on HPV-DNA markers and failed to investigate the expression of $\mathrm{P} 16^{\mathrm{INK} 4 \mathrm{a}}$ a surrogate marker of oncogenic HPV infection. P16 ${ }^{\text {INK4a }}$ is a tumor suppressor protein that is downregulated in many tumor types [17], but overexpressed in HPV-related tumors [18]. Very little is known on the possible co-carcinogenic role of EBV and other herpesviruses in HNSCC. We thus evaluated the prevalence of the above agents and their possible co-infection rate in a series of Italian SCC cases.

\section{Material and methods}

\section{Tissue samples}

Archived formalin-fixed, paraffin-embedded (FFPE) tissue blocks were collected for 51 SCC cases. FFPE sections were obtained from the archive of the Pathology Department. Tissue specimens were obtained from patients with HNSCC (oral cavity and oropharynx) before initiation of radiotherapy, while samples of laryngeal HNSCC were taken at surgery. Eligible cases included newly diagnosed primary HNSCC. At the time of sampling, patients had not received antitumor therapy. The study was approved by the Ethics Committee of IRCCS San Martino IST (Genoa, Italy). As archived tissue was used, no written consent was required.

$5 \times 10 \mu \mathrm{m}$ sections were removed from the blocks were deparaffinized with xylene and rehydrated through a graded series of distilled water-ethanol solutions. QIAamp DNA FFPE Tissue Kit (QIAGEN, Hilden, Germany) was used for DNA extraction. DNA was quantified with a NanoDrop 3300 fluorospectrometer using the RiboGreen dye diluted in TE buffer (Thermo Fisher Scientific, Monza, Italy).

\section{Detection of human herpesviruses (HHV) and HPV genotypes}

Tissue sections were retrospectively analyzed for EBV and other HHV (CMV, HHV-6, $-7,-8$ ) by quantitative real-time PCR assays [19]. HPV detection and genotyping was performed using a multiplex real-time PCRs based on hybridization-fluorescence detection of amplified products (PANA RealTyperTM HPV assay - Panagene; DBA Italia, Milano, Italy). The assay was performed for detection and differentiation of 20 high-risk HPV types $(16,18,26,31$, $33,35,39,45,51,52,53,56,58,59,66,68,69,70,73$, and 82 ), two low-risk genotypes ( 6 and 11$)$ from clinical specimens. Eighteen genotypes (30, 32, 34, 40, 42, 43, 44, 54, $55,61,62,67,74,81,83,84,87$, and 90 ) are detected, but not typed.

\section{Immunohistochemistry (IHC) for p16 $16^{\text {INK4A }}$}

Immunoperoxidase staining was done on $4 \mu \mathrm{m}$ sections of FFPE tissue using the LSAB2 horseradish peroxidase system (DAKO, Carpentaria, CA). Antigen retrieval was done by microwave heating for $10 \mathrm{~min}$ in $10 \mathrm{mM}$ citrate buffer (pH 6.0). A p16 ${ }^{\mathrm{INK} 4 \mathrm{~A}}$ monoclonal antibody (Novocastra-Leica, Buccinasco, Italy) was used at 1:50 dilution. Cases were classified as either positive (with cut-off value of stained nuclei $>40 \%$ ) or negative.

\section{Statistical analysis}

Statistical analysis was performed for virus positivity, p $16^{\mathrm{INK} 4 \mathrm{~A}}$ positivity, clinical and demographic data using the Prism GraphPad software (San Diego, CA). Statistical significance was defined as $p<0.05$.

\section{Results}

The study included 51 patients, 40 with oropharyngeal cancer (OPSCC: 5 in pharinge, 27 in larynge, 5 in vocal cord, 3 in base of tongue) and 11 with oral cancer (OSCC: 7 in floor of the mouth, 3 in buccal mucose, 1 in tongue). Demographic and clinical data were also recovered. Mean age was 65 years (standard deviation \pm 14 years) ranging from 43 to 94 years. Males (69\%) with smoking $(70 \%)$ and alcohol abuse $(60 \%)$ problems were prevailing in the series. All cases resulted negative for HHV-6, -7 and -8 . CMV was detected in only two cases (mobile tongue SCC and epiglottis SCC).

Overall, of the 51 patients, 10 cases (19.6\%) were positive for HPV (9 HPV-16 and 1 HPV-45) and 12 (23.5) for $\mathrm{p} 16^{\mathrm{INK} 4 \mathrm{~A}}$. . Two patients had $\mathrm{p} 16^{\mathrm{INK} 4 \mathrm{~A}}$ positive and negative HPV PCR.

The prevalence of HPV and EBV, according to SCC primary site and hystotype and is presented in Table 1. In particular, the prevalence of EBV resulted significantly 
Table 1 Expression of HPV-induced p1 $6^{\text {INK4A }}$ and prevalence of HPV and EBV in squamous cell carcinoma according to primary tumor site and histotype

\begin{tabular}{llllc}
\hline & $\begin{array}{l}\text { HPV-induced } \\
\text { p16 }\end{array}$ & $\begin{array}{l}\mathrm{HPV}^{+} \\
(\%)\end{array}$ & $\begin{array}{l}\mathrm{EBV}^{+} \\
(\%)\end{array}$ & $\begin{array}{l}\text { Co-infection } \\
\text { HPV-EBV (\%) }\end{array}$ \\
\hline $\begin{array}{l}\text { Primary site } \\
\text { (No.) }\end{array}$ & & & & \\
OPSCC (40) & $11(27.5)$ & $10(25)$ & $18(45)$ & $4(10)$ \\
OSCC (11) & $1(9.1)$ & - & $8(72.7)$ & \\
Histotype (No.) & & & & \\
NKSCC (26) & $11(42.3)$ & 9 & 16 & $4(15.4)$ \\
KSCC (25) & $1(4)$ & $(34.6)$ & $(61.5)$ & \\
& & $1(4)$ & 10 & \\
\hline
\end{tabular}

KSCC keratinizing squamous cell carcinoma, NKSCC nonkeratinizing squamous cell carcinoma. HPV p16 detected by immunohistochemistry (IHC)

higher compared to that of HPV (EBV 51\% vs. HPV $19.6 \% ; P=0.05)$. Regarding the subsites of the head and neck region, HPV infection was found in 25\% of OPSCC and in none of the OSCC. Conversely, EBV infection was found in $72.7 \%$ of OSCC and $45 \%$.of OPSCC cancer cases (Table 1) HPV and EBV co-infection was detected in $4(10 \%)$ OPSCC. The prevalence of HPV but no EBV was associated with the non-keratinizing SCC type (NKSCC) compared to the keratinizing SCC type $($ KSCC) $($ HPV-DNA $P<0.005$; EBV $=0.054)$.

Of note, all 9 NKSCC cases HPV positive were also p $16^{\mathrm{INK} 4 \mathrm{~A}}$-positive. In contrast, only $1 / 15 \mathrm{KSCC}$ cases was HPV positive and also $\mathrm{p} 16^{\mathrm{INK} 4 \mathrm{~A}}$-positive. The data indicate that NKSCC cases are more likey $\mathrm{p} 16^{\mathrm{INK} 4 \mathrm{~A}}$ - and HPVpositive than KSCC ( $P=0.0001$ and $P<0.05$, respectively). In Fig. 1 is showed HPV-induced p16 ${ }^{\mathrm{INK} 4 \mathrm{~A}}$-positive NK SCC at the base of tongue and in laryngeal. For EBV, although there was no difference in histopathological type, NKSCC cases were also more frequently EBV-positive compared to KSCC cases (data not shown).

\section{Discussion}

We explored the prevalence of HPV and potentially oncogenic herpesviruses (HHVs) in archival tissue of SCC cases from different anatomic sites. This is the first study from Italy reporting on the HPV and EBV co-infection in cancers.

The overall HPV prevalence in HNSCC was low for OPSCC (25\%) and absent in OSCC compared to the reported global prevalence of HPV in the same types of cancer (45.8\% OPSCC, $24.2 \%$ OSCC, respectively) $[9,10,18]$ but in accordance with one Italian study (OPSCC 27\%) [11].

Among the $12 \mathrm{HPV}$ types defined as carcinogenic in cervical carcinoma by the International Agency for Research on Cancer (types 16, 18, 31, 33, 35, 39, 45, 51, 52, 56,58 and 59), only type 16 appears to play an

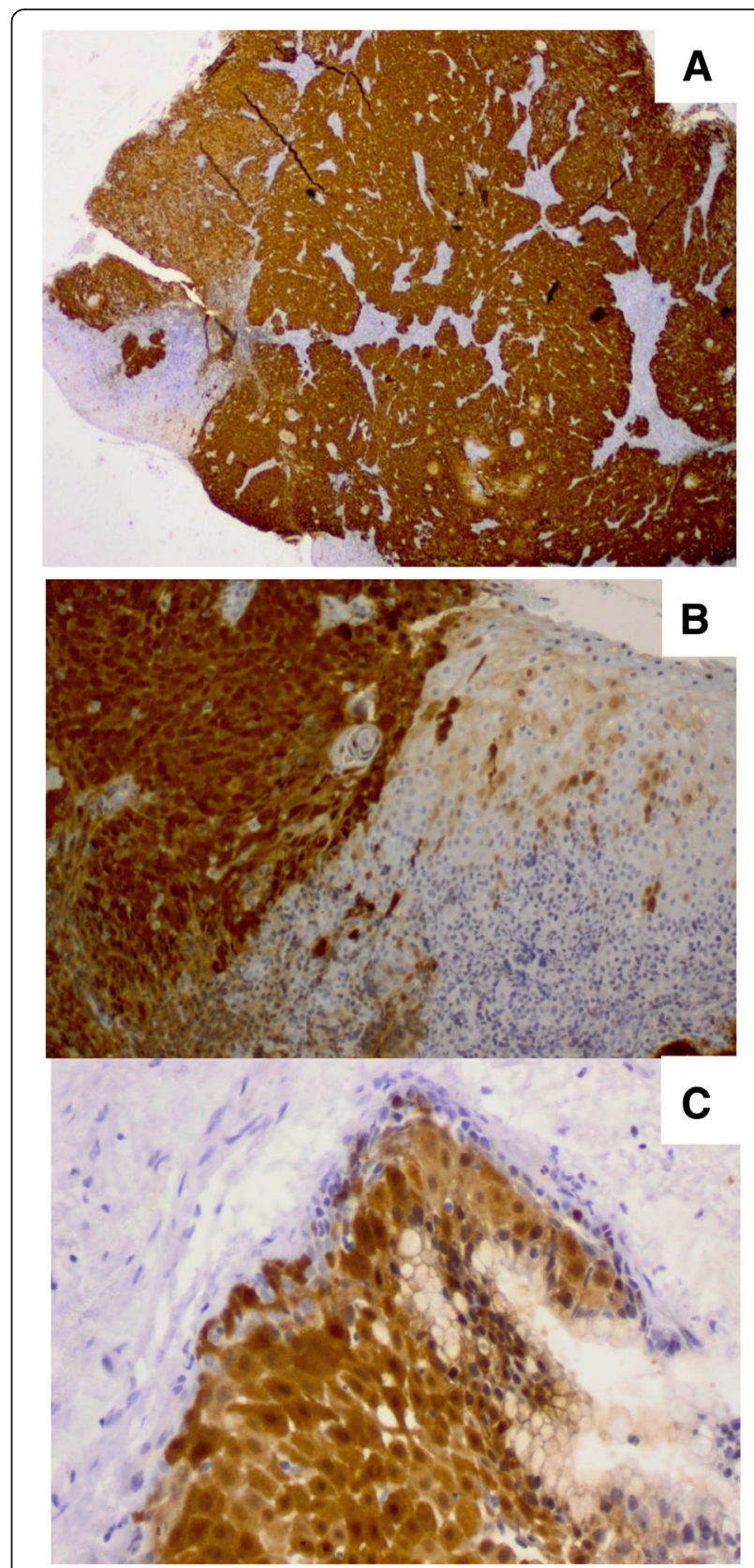

Fig. 1 Expression of HPV-induced p16INK4A in squamous cell carcinoma (SCC). (a-b) p1 $6^{\text {INK4A }}$-positive section of non-keratinizing SCC at the base of tongue ( $2 x$ and 10X magnification); (c) p16 $6^{\text {INK4A }}$ positivity in laryngeal SCC (10X magnification)

oncogenic role in OPSCC [9]. Among HPV-positive OPSCC cases, HPV16 was the most prevalent genotype (9 of 10 cases)., HPV-45 DNA was detected in 1 case suggesting an oncogenic role of this genotype in OPSCC as well as cervical carcinoma.

The detection of HPV DNA alone is not sufficient to determine tumor causality. For cervical carcinoma and for oropharyngeal and laryngeal cancer the co-expression 
of several markers (alone or in combination) is required to discriminate HPV-driven from non-HPV-driven cases [20].

The $\mathrm{p} 16^{\mathrm{INK4a}}$ protein is considered a surrogate marker of HPV infection in SCC of the cervix and head and neck, as well as in HPV-associated anogenital cancer [21]. In HNSCC, p16 ${ }^{\text {INK4a }}$ appears as a reliable marker in tumors arising from the oropharynx, larynx, and oral cavity [21, 22]. A portion of oropharyngeal tumors show upregulated $\mathrm{p} 16^{\mathrm{INK} 4 \mathrm{a}}$, but lack HPV positivity.

In our series, infection with high-risk HPV genotypes was not significantly associated with oral cancers. This is in line with what reported [4]. In contrast, a strong association of high-risk HPV genotypes with oral cancer has been observed by others [3].

We classified SCC histotypes into two groups: nonkeratinizing and keratinizing SCC. In the investigated series, NKSCC was strongly associated with high-risk HPV genotypes and $\mathrm{p} 16^{\mathrm{INK} 4 \mathrm{~A}}$ positivity ( $69 \%$ and $100 \%$, respectively) compared to KSCC (8\% and 36\%, respectively). Of interest is the frequent detection of HPV and EBV co-infection in oral and oropharyngeal cancer among Italian patients. HPV infection is considered a sexually-transmitted infection, while EBV is mostly transmitted through saliva [23]. Both agents are capable of establishing long-term infection in epithelial cells. A variety of stimuli do play a role in activating viral genome expression [23]. Chemicals and sexual behaviors can play a role. The results of our study confirm the low prevalence of HPV- driven HNSCC in Italy, in line with data from other countries of southern Europe. HPV16 appears to play a role mostly in the oropharynx, while EBV is more prevalent in the oral cavity cancer. Thus, current HPV vaccines [24] may be expected to prevent part of head and neck cancer, but an EBV vaccine remains badly needed [23].

\section{Abbreviations}

CMV: cytomegalovirus; EBV: Epstein-Barr virus; FFPE: formalin-fixed paraffinembedded; HHVs: human herpesviruses; HNSCC: head and neck squamous cell carcinoma; HPV: Human Papilloma Virus; OPSCC: oropharyngeal squamous cell carcinoma; OSCC: oral squamous cell carcinoma; QPCR: quantitative Real-time PCR

\section{Acknowledgements}

We thank for excellent technical assistance Roberta Giorgini, Roberta Schiavone, Franco Vigo, Angela Massarini, Massimo Larosa and Paolo Caprari who performed histopathological analysis of archival tissues. We are grateful to Dr. Eleonora Indemini for recovering clinical data.

\section{Funding}

Research funded by the Fondo di Ateneo Quota Competitiva - University of Milano-Bicocca, grant N²016-ATESP-0032 to FB.

\section{Availability of data and materials}

The datasets used and/or analyzed during the current study are available from the corresponding author on request.

\section{Authors' contributions}

All authors collaborated in the study design; FB, AT, participated in diagnosing and managing of cases; FB and AR performed QPCR; LA performed ICH and discussed results; GC and FD extracted and analyzed clinical data; FB and AT participated in data analysis, interpretation, manuscript preparation and revision. All authors read and approved the final manuscript.

Ethics approval and consent to participate

None required.

\section{Consent for publication}

Not applicable.

\section{Competing interests}

The authors declare that they have no competing financial or non-financial interests.

\section{Publisher's Note}

Springer Nature remains neutral with regard to jurisdictional claims in published maps and institutional affiliations.

\section{Author details}

${ }^{1}$ Department of Medicine and Surgery (School of Medicine), University of Milano-Bicocca, Monza, Italy. ${ }^{2}$ DISSAL Department of Dermatology, IRCCS A.O.U. San Martino-IST, Genoa, Italy. ${ }^{3}$ Laboratory of Medical Microbiology, Department of Biotechnology and Life Sciences, University of Insubria, Varese, Italy. ${ }^{4}$ Department of Pathology, ASL 3 Genovese, Genoa, Sampierdarena, Italy. ${ }^{5}$ Laboratory of Medical Microbiology, University of Milano-Bicocca, Via Cadore 48, 20900 Monza, Italy.

Received: 28 June 2018 Accepted: 23 October 2018

Published online: 09 November 2018

\section{References}

1. Ferlay J, Steliarova-Foucherand E, Lortet-Tieulentetal J. Cancerincidence and Mortality patterns in Europe: estimates for 40 countries in 2012. Eur J Cancer. 2013;49:1374-403.

2. Herrero R, Castelselsague X, Pawlita M, et al. Human papillomavirus and oral cancer: the international agency for research on cancer multicenter study. J Natl Cancer Inst. 2003;95:1772-8.

3. Gillison ML, Koch WM, Capone RB, et al. Evidence for a causal association between human papillomavirus and a subset of head and neck cancers. J Natl Cancer Inst. 2000;92:709-20.

4. Smith EM, Rubenstein LM, et al. Complex etiology underlies risk and survival in head and neck cancer human papillomavirus, tobacco, and alcohol: a case for multifactor disease. J Oncol. 2012:571862.

5. Mork J, Lie AK, Glattre E, et al. Human papillomavirus infection as a risk factor for squamous-cell carcinoma of the head and neck. N Engl J Med. 2001;344:1125-31.

6. D'Souza G, Kreimer AR, Viscidi R, et al. Case-control study of human papillomavirus and oropharyngeal cancer. N Engl J Med. 2007;356:1944-56.

7. Heck JE, Berthiller J, Vaccarella S, et al. Sexual behaviours and the risk of head and neck cancers: a pooled analysis in the international head and neck Cancer epidemiology (INHANCE) consortium. Int J Epidemiol. 2009;39:166-81

8. Nair S, Pillai MR. Human papillomavirus and disease mechanisms: relevance to oral and cervical cancers. Oral Dis. 2005:11:350-9.

9. Kreimer AR, Clifford GM, Boyle P, et al. Human papillomavirus types in head and neck squamous cell carcinomas worldwide: a systematic review. Cancer Epidemiol Biomark Prev. 2005;4:467-75.

10. Abogunrin S, Di Tanna GL, Keeping S, et al. Prevalence of human papillomavirus in head and neck cancers in European populations: a metaanalysis. BMC Cancer. 2014;14:968.

11. Baboci L, Holzinger D, Boscolo-Rizzo P, et al. Low prevalence of HPV-driven head and neck squamous cell carcinoma in North-East Italy. Papillomavirus Res. 2016;2:133-40.

12. Boscolo-Rizzo P, Zorzi M, Del Mistro A, et al. The evolution of the epidemiological landscape of head and neck cancer in Italy: Is there evidence for an increase in the incidence of potentially HPV-related carcinomas. PLoS One. 2018;13(2):e0192621.

13. Longnecker R, Kieff E, Cohen Jl. Epstein Barr Virus. In: Knipe DM, Howley PM, editors. Fields Virology. 6th ed. Philadelphia: Lippincott Williams \& Wilkins; 2013. p. 11959. 
14. She $Y$, Nong $X$, Zhang $M$, Wang $M$. Epstein-Barr virus infection and oral squamous cell carcinoma risk: A meta-analysis. PLoS One 2017;24:12(10): e0186860.

15. Cruz I, Van Den Brule AJ, Brink AA, et al. No direct role for Epstein-Barr virus in oral carcinogenesis: a study at the DNA. RNA and protein levels Int J Cancer. 2000;86(3):356-61.

16. Alibek K, Kakpenova A, Yeldar Baiken Y. Role of infectious agents in the carcinogenesis of brain and head and neck cancers. Infect Agent Cancer. 2013;8:7-16. https://doi.org/10.1186/1750-9378-8-7.

17. Romagosa C, Simonetti S, López-Vicente L, et al. p16(Ink4a) overexpression in cancer: a tumor suppressor gene associated with senescence and highgrade tumors. Oncogene 2011;30(18):2087-2097.

18. Ndiaye C, Mena M, Alemany L, et al. HPV DNA, E6/E7 mRNA, and p16INK4a detection in head and neck cancers: a systematic review and meta-analysis. Lancet Oncol. 2014;15(12):1319-31.

19. Broccolo F, Chiari S, Piana A, et al. Prevalence and viral load of oncogenic human papillomavirus types associated with cervical carcinoma in a population of North Italy. J Med Virol. 2009;81(2):278-87.

20. Pannone G, Rodolico V, Santoro A, et al. Evaluation of a combined triple method to detect causative HPV in oral and oropharyngeal squamous cell carcinomas: P16 immunohistochemistry, consensus PCR HPV-DNA, and in situ hybridization, infect. Agent Cancer. 2012;7.

21. Donà MG, Spriano G, Pichi B, et al. M. Human papillomavirus infection and p16 overexpression in oropharyngeal squamous cell carcinoma: a case series from 2010 to 2014. Future Microbiol. 2015;10(8):1283-91.

22. Albers AE, Qian X, Kaufmann AM, et al. Meta analysis: HPV and p16 pattern determines survival in patients with HNSCC and identifies potential new biologic subtype. Sci Rep. 2017;7(1):16715.

23. Dasari V, Bhatt KH, Smith C, Khanna R. Designing an effective vaccine to prevent Epstein-Barr virus-associated diseases: challenges and opportunities. Expert Rev Vaccines. 2017;16(4):377-90.

24. Wang C, Dickie J, Sutavani RV, Pointer C, Thomas GJ, Savelyeva N. Targeting Head and Neck Cancer by vaccination. Front Immunol 2018; 23: (9) 830. Review.

Ready to submit your research? Choose BMC and benefit from:

- fast, convenient online submission

- thorough peer review by experienced researchers in your field

- rapid publication on acceptance

- support for research data, including large and complex data types

- gold Open Access which fosters wider collaboration and increased citations

- maximum visibility for your research: over $100 \mathrm{M}$ website views per year

At $\mathrm{BMC}$, research is always in progress.

Learn more biomedcentral.com/submissions 